\title{
Vascular bioactivity of anthocyanin degradants: inhibition of endothelial superoxide production
}

\author{
M. Edwards, C. Czank, A. Cassidy and C. D. Kay \\ Department of Nutrition, Norwich Medical School, University of East Anglia, Norwich, NR4 7TJ, UK
}

\begin{abstract}
Anthocyanins, a sub-class of the flavonoid family of phenolic phytochemicals, are reported to have significant vasoprotective activity ${ }^{(1)}$, but their low bioavailability suggests bioactivity may be mediated by their degradation products or metabolites ${ }^{(2)}$. The present study aimed to investigate the vascular bioactivity of selected anthocyanins and phenolic degradants, and elucidate their potential molecular mechanisms of action. Bioactivity was assessed by screening for the modulation of superoxide-generating NADPH oxidase (NOX) and endothelial nitric oxide synthase (eNOS) activity in human umbilical vein endothelial cells.

Angiotensin II (Ang II)-stimulated superoxide production was quantified by reduction of ferricytochrome c, based on previously reported methods ${ }^{(3)}$, and commercially available kits were used to quantify eNOS expression (Quantikine, R\&D Systems, UK) and nitric oxide (NO) production (Nitrite/Nitrate Assay, Cayman Chemical Company, USA). Angiotensin II- or tumour necrosis factor-alpha $(\mathrm{TNF}-\alpha)$-stimulated expression of NOX isoform/subunit mRNA and/or protein was assessed by reverse transcription - quantitative polymerase chain reaction and immunoblotting.

Superoxide production was significantly decreased $(P<0.05$; ANOVA with Tukey post-hoc; $n=3)$ by the anthocyanins peonidin-3glucoside, petunidin-3-glucoside and malvidin-3-glucoside; and the anthocyanin phenolic degradants protocatechuic acid, vanillic acid, and syringic acid. Cyanidin-3-glucoside, peonidin-3-glucoside, and petunidin-3-glucoside also significantly upregulated eNOS $(P<0.05$; $n=3$ ), whereas the anthocyanin degradants had no effect. Based upon these bioactivity data, the phenolic degradant vanillic acid was selected to explore molecular mechanisms potentially underlying the observed bioactivity. Here, vanillic acid (at $0.1-10 \mu \mathrm{M})$ did not significantly $(P>0.05)$ modulate mRNA levels of the NOX4 isoform (Figure $1 \mathrm{~A})$ or the associated integral membrane protein p22 $2^{\text {phox }}$ (Figure 1B); nor did vanillic acid significantly affect protein levels of the NOX2 \& 4 isoforms (data not shown) or p47 $7^{\text {phox }}$, a key NOX2 subunit (Figure 1C). Trends were observed towards an upregulation of p22 ${ }^{\text {phox }}$ mRNA $(P=0.12$ at $1 \mu \mathrm{M})$, and a decrease in p47 $7^{\text {phox }}$ protein $(P=0.27$ at $10 \mu \mathrm{M})$.
\end{abstract}
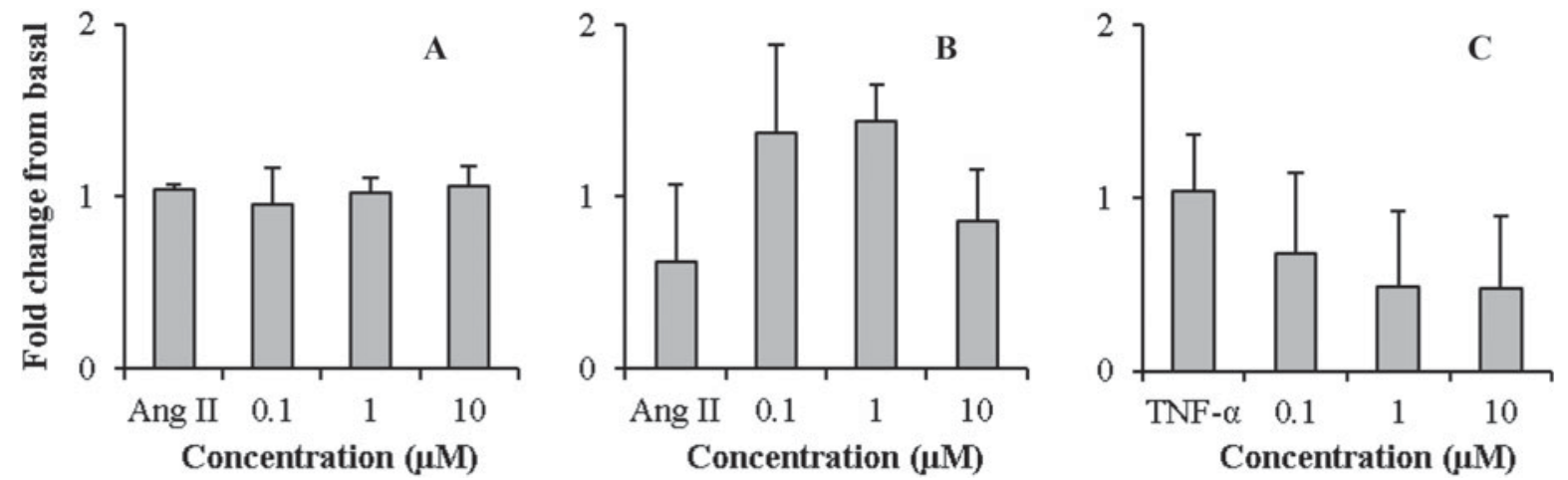

Fig. 1. NOX4 (A) and p22 $2^{\text {phox }}\left(\right.$ B) mRNA; $47^{\text {phox }}$ protein (C). Data graphed as mean $+\mathrm{sD}, n=3(\mathrm{~A}, \mathrm{~B})$ or 4 (C).

In conclusion, anthocyanin phenolic degradants could enhance vascular function in vivo by decreasing superoxide production and thus NO scavenging, as opposed to direct stimulation of NO production. Vanillic acid may inhibit endothelial superoxide production by the NOX2 isoform through downregulation of the key cytosolic subunit $\mathrm{p} 47^{\text {phox }}$, and this mechanism should be further explored and confirmed in human arterial endothelial cells.

This work was supported by a BBSRC-CASE studentship (M. Edwards) in partnership with GlaxoSmithKline plc. (United Kingdom).

1. Erdman JW, Jr., Balentine D, Arab L et al. (2007) J Nutr 137, 718S-37S.

2. Williamson G \& Clifford MN (2010) Br J Nutr 104, S48-S66.

3. Steffen Y, Gruber C, Schewe T et al. (2008) Arch Biochem Biophys 469, 209-19. 